\title{
Magnetic pulse processing of strawberries in the climatic chamber
}

\author{
Dmitriy Khort ${ }^{1}$, Alexey Kutyrev ${ }^{1, *}$, Rostislav Filippov ${ }^{1}$, and Stepan Semichev ${ }^{1}$ \\ ${ }^{1}$ Federal Scientific Agroengineering Center VIM, 1-st Institutsky proezd, 5, Moscow, 109428, Russia
}

\begin{abstract}
The article presents the results of experimental studies on the magnetic pulse treatment of strawberries in a climatic chamber. The analysis of the obtained data showed that the greatest effect from the treatment of plants with a low-frequency magnetic pulse field in the phase of peduncle formation was obtained on plants that were treated with a magnetic induction equal to $4 \mathrm{MT}$, a pulse repetition frequency of $32 \mathrm{~Hz}$ and a duty cycle of 20 . The relationship between the parameters of low-frequency magnetic pulse radiation and the photosynthetic activity of the leaf apparatus at various stages of ontogenesis, and the qualitative composition of fruits at the stage of maturation is revealed. The high sensitivity of plant organisms to the effects of energy factors, the parameters of which differ in exposure and physical factors, is shown. At the same time, the nature of the response of plants is complex, ambiguous and is determined not only by the electrophysical parameters of the electric field, but also by the specific, varietal and technological features of the crop.
\end{abstract}

\section{Introduction}

In the process of evolution, wildlife used the natural electromagnetic fields of the external environment as a source of information that ensures the continuous adaptation of organisms to changes in various environmental factors. Based on this, it is possible to explain the high sensitivity of organisms to changes in the electromagnetic field that carry information signals [1-3]. Organisms can distinguish the intensity of the magnetic field and sense the direction in which the magnetic lines of force pass through their body. Moreover, magnetic fields with a strength close to the earth's geomagnetic field have the maximum efficiency [4-6]. Strong deviations of the electromagnetic field from the natural level to a greater or lesser extent, go beyond the limits of the optimal life activity of living organisms and are a stressful factor. With full screening, the rate of reproduction of microorganisms changes, there is a violation of metabolic processes, changes in the morphology and various functions of a living organism [7-10]. In some cases, the most significant biological effect can be expected when a resonance occurs, when one or more parameters of external influences are close to the parameters of biosystems [11, $12]$.

The effect of a magnetic field on a living organism is determined by such parameters as intensity (field strength, magnetic induction), gradient (rate of rise or fall of the field), vector (direction of field lines of force), exposure (duration of exposure), frequency (number of magnetic field oscillations per second), pulse shape (characteristic of rise and fall of intensity), localization (spatial characteristic) [13].

In this regard, there is an urgent task to expand the field of application of low-frequency magnetic fields and to establish parameters and develop special technical means for magnetic pulse treatment of plants, allowing to reduce the use of pesticides, increase the volume of production and product quality.

\section{Materials and methods}

Magnetic-pulse processing was performed using the developed automated MPP (magnetic pulse processing) apparatus [14, 15] (Fig. 1).

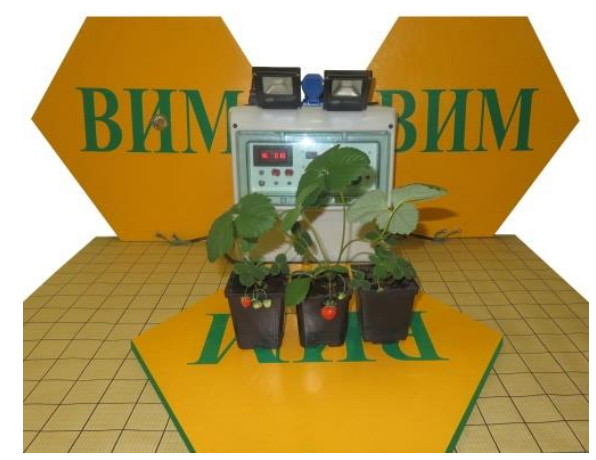

Fig. 1. Automated magnetic pulse processing machine.

For experimental studies in the conditions of the climatic chamber, young rosettes of plants (stolons) of strawberry (Fragaria vesca L.) "Malvina" were used, which were planted in vessels filled with a soil substrate

\footnotetext{
* Corresponding author: alexeykutyrev@gmail.com
} 
with a volume of 5 liters. 5 plants were placed in each vessel. Each experiment was repeated 2 times (Fig. 2).

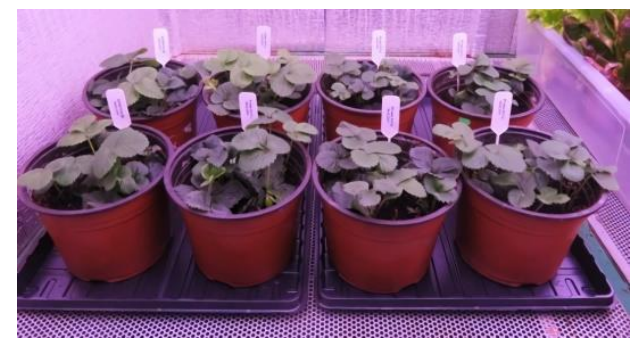

Fig. 2. 30-day-old strawberry plants treated with a lowfrequency pulsed magnetic field.

As a control, plants that were not treated with a lowfrequency magnetic pulse field were used. After processing, the strawberry plants were placed in a climatic chamber, in which they were grown at a temperature of $23-25^{\circ} \mathrm{C}$, with a relative humidity of 50 $60 \%$ during the entire growing season. The MPP modes are presented in Table 1.

Table 1. Magnetic pulse processing modes used in the experiment.

\begin{tabular}{|c|c|c|c|}
\hline $\begin{array}{c}\text { Mode } \\
\text { № }\end{array}$ & $\begin{array}{c}\text { Frequency, } \\
\mathbf{H z}\end{array}$ & Duty cycle & $\begin{array}{c}\text { Induction, } \\
\mathbf{m T}\end{array}$ \\
\hline 1 & 32 & 20 & 6 \\
\hline 2 & 8 & 20 & 6 \\
\hline 3 & 32 & 12 & 6 \\
\hline 4 & 8 & 12 & 6 \\
\hline 5 & 32 & 20 & 4 \\
\hline 6 & 8 & 16 & 4 \\
\hline 7 & 32 & 12 & 4 \\
\hline 8 & 8 & 12 & 4 \\
\hline 9 & \multicolumn{3}{|c}{ Control } \\
\hline
\end{tabular}

To determine the morphophysiological parameters, 5 strawberry leaves were selected from each vessel and the following parameters were determined: the dry and wet mass of the leaves, their area, and the content of photosynthetic pigments. Evaluation of the effect of the specified treatment modes on the growth and development of strawberry plants was carried out by analyzing samples of vegetative organs (leaves) of plants 3 weeks after their treatment. The following biometric indicators and indicators of photosynthetic activity of strawberry plants were determined: leaf area, dry and raw leaf mass, content of photosynthetic pigments (chlorophyll a, b, and carotenoids). Determination of the quality indicators of fruits (sugar content, ascorbic acid, nitrate accumulation, osmotic pressure of cell juice) was carried out at the stage of fruit maturation by wet chemistry. The results of the analyses are presented in the form of tables and histograms. Each mode includes three parameters: magnetic induction $(\mathrm{mT})$, pulse repetition rate $(\mathrm{Hz})$, duty cycle. The analysis of fruits was carried out according to the following main indicators: sucrose concentration, ascorbic acid content, nitrate content, $\mathrm{mg} / \mathrm{kg}$, osmotic pressure in the cell.

\section{Results and discussions}

The results of the evaluation of the effect of magnetic pulse treatment on the mass and leaf area of the selected samples (1-9) of strawberry plants in the phase of formation (extension) of peduncles (30 days after processing) and in the phase of fruit maturation are shown in Figures 3,4.

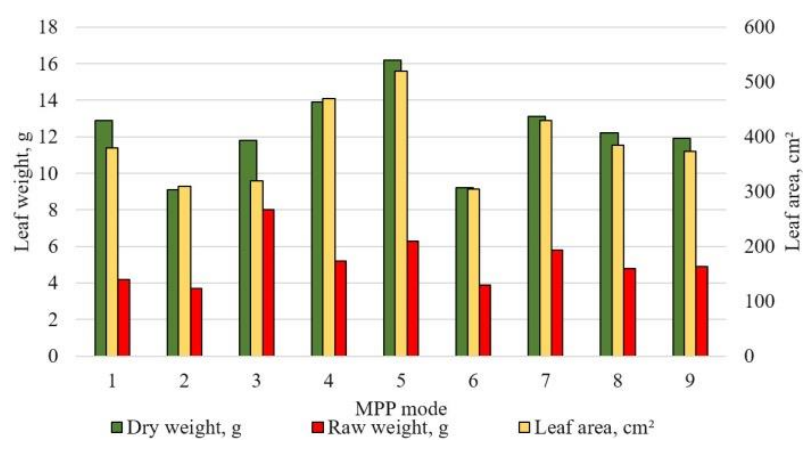

Fig. 3. Influence of different modes of magnetic-pulse treatment of plants on the mass and area of leaves of strawberry of the garden variety "Malvina" in the phase of flower stalks release.

Analysis of the data showed that the greatest effect of the treatment plants low frequency magnetic field pulse in the phase formation of stems was obtained on the sample No. 5, plants which were treated magnetic induction, equal to $4 \mathrm{MT}$, the pulse frequency of $32 \mathrm{~Hz}$ and a duty cycle of 20 (mode No. 5). In this treatment mode, the positive values of deviations $(\Delta, \%)$ of biometric indicators of plant morphological organs from the control were: the raw mass of plants is $39.6 \%$, the dry mass is $26.5 \%$, and the leaf area is $42.0 \%$.

A similar pattern of the influence of magnetic pulse treatment of strawberry plants was observed when analyzing biometric indicators of the mass and area of leaves in the fruit ripening phase (Fig. 4).

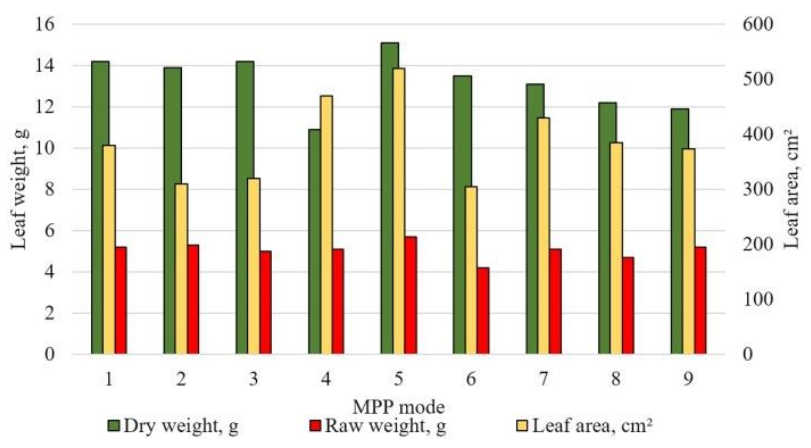

Fig. 4. The effect of different MPP regimes on the mass and area of leaves of strawberry in the fruit ripening stage.

The greatest values of deviations of the mass and area of the leaves from the control values in the maturation phase were also obtained in the mode No. 5 . The values of deviations of the received data from the control indicators in the specified processing mode were as follows: The raw mass of plants is $12.2 \%$, the dry mass is $13.6 \%$, and the leaf area is $14.8 \%$, which is evidence of the preservation of the positive stimulating 
effect obtained by plants at the stage of peduncle formation.

The effect of different modes of magnetic pulse treatment on the photosynthetic activity of plants at different stages of development was assessed by the content of chlorophyll a, b and carotenoids in the leaves. The results of determining the content of pigments in the leaves of plants in the phase of extension of the peduncles are shown in Figures 5,6.

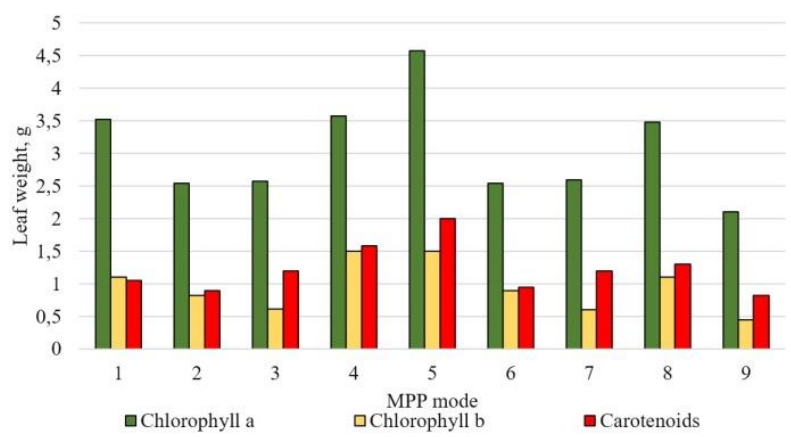

Fig. 5. The content of photosynthetic pigments in the leaves of strawberry garden variety "Malvina" in the phase of extension of peduncles.

The analysis of the obtained results showed a significant positive effect of all the modes of magnetic pulse treatment of strawberry plants used. Thus, the values of deviations of the content of chlorophyll a, b and carotenoids in plant leaves from the control parameters varied depending on the treatment mode: chlorophyll a from 30.6 to $131.6 \%$, chlorophyll $\mathrm{b}$ from 39.4 to $189.7 \%$, carotenoids from 20.4 to $148.2 \%$, but the greatest stimulating effect of exposure to plants, increasing their photosynthetic activity by almost 1.5 times compared to the control, was obtained in treatment mode № 5 .

The results of determination of pigment content in the leaves of plants in the phase of fruit ripening showed that the pigment content in the leaves of plants treated with various modes was significantly lower benchmarks, which is associated with the biological reduction process photosynthetic activity of leaf apparatus in the phase of fruit ripening (Fig.6).

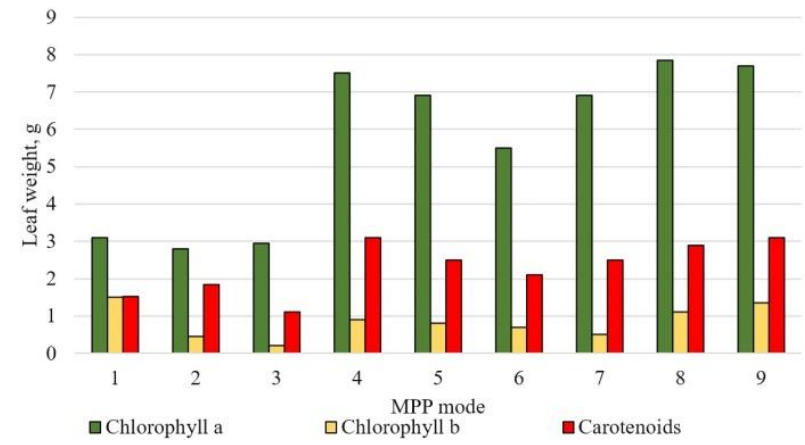

Fig. 6. Influence of the modes of magnetic-pulse treatment of plants on the photosynthetic activity of the vegetative organs of strawberries (leaves) in the fruit-ripening phase.

The analysis of the quality indicators of fruits showed that the studied modes of magnetic-pulse treatment of plants in the phase before the formation of flower stalks have an ambiguous effect on the quality indicators of strawberry fruits. It is not possible to establish a single optimal mode of magnetic pulse treatment of plants, which has a stimulating effect on the quality of fruits. Therefore, the influence of the used modes of magnetic pulse treatment of plants on the content of each qualitative indicator was evaluated.

Figure 7 shows a histogram of changes in the concentration of sucrose in strawberry fruits at the stage of their maturation, depending on the modes of lowfrequency magnetic pulse treatment of plants. The figure shows that an increase in the sucrose content in comparison with the control in strawberry fruits was observed at magnetic induction values of $6 \mathrm{mT}$ (modes $1,2,3,4)$.

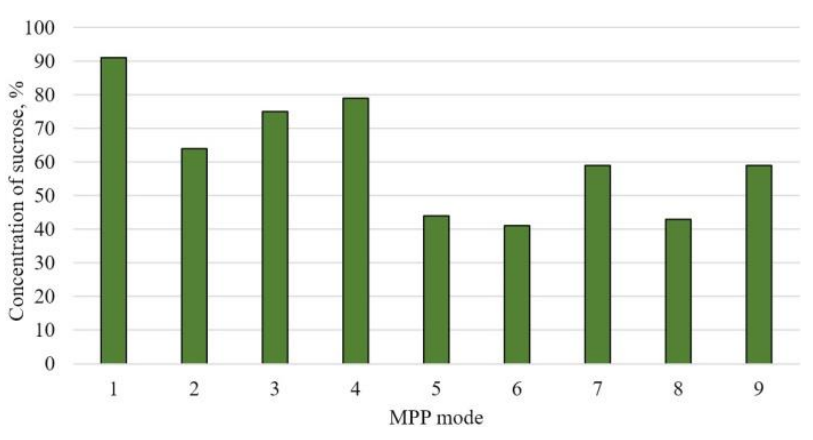

Fig. 7. Changes in the concentration of sucrose in strawberry fruits.

The greatest stimulating effect of low-frequency magnetic pulse treatment of plants was obtained in the mode No. 1, the parameters of which were: magnetic induction $\mathrm{B}=6 \mathrm{mT}$; pulse repetition frequency $\mathrm{Fs}=32$ $\mathrm{Hz}$; duty cycle $\mathrm{S}=20$, in which the content of sucrose in strawberries increased by $32 \%$ compared to the control. When treating plants with modes with induction of 4 $\mathrm{mT}$, no stimulating effect on the accumulation of sucrose was found.

Figure 8 shows the impact of different modes of magnetic-pulse treatment of strawberry plants in the phase of peduncle formation on the content of ascorbic acid in berries.

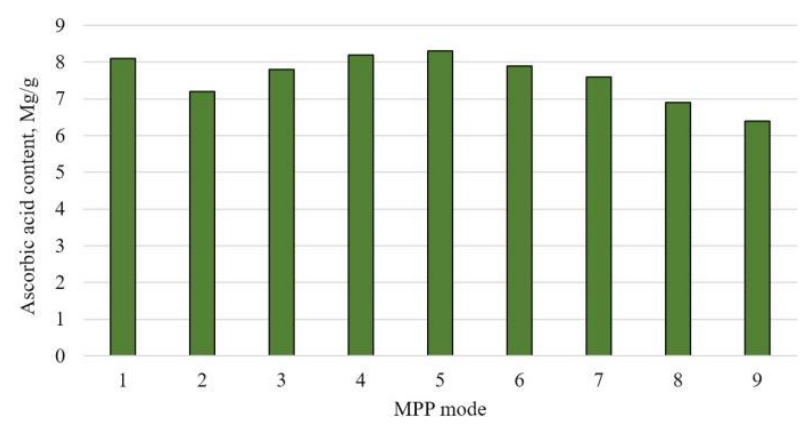

Fig. 8. The content of ascorbic acid in the fruits of strawberries in the fruit ripening phase.

It was noted that in all the plant treatment modes used, an increase in ascorbic acid in berries was observed from $10 \%$ to $25.7 \%$ compared to the control indicator. However, the greatest stimulating effect was 
obtained when the plants were treated with mode 5 with the induction of a magnetic field of $4 \mathrm{mT}$, pulse repetition frequency of $32 \mathrm{~Hz}$ and duty cycle of 20 , in which the content of ascorbic acid in the fruits increased by $25.7 \%$ compared to the control. A positive effect on the accumulation of ascorbic acid in the fruits of modes No. 1 and 4 was established, when exposed to which an increase in ascorbic acid in the fruits was noted by $21.4 \%$ and $22.8 \%$ (induction $\mathrm{B}=6 \mathrm{mT}$, frequency $\mathrm{Fs}=$ $32 \mathrm{~Hz}$, duty cycle $\mathrm{S}=20$ and 12 , respectively).

The content of nitrates in the presented samples of strawberry fruits ranged from 27.0 to $33.0 \mathrm{mg} / \mathrm{kg}$, which does not exceed the maximum permissible concentrations. The highest values of nitrate accumulation in berries (12.2\% compared to the control) were obtained with low-frequency magnetic pulse treatment of plants with mode 5 (induction $\mathrm{B}=4 \mathrm{mT}$, frequency $\mathrm{Fs}=32 \mathrm{~Hz}$, duty cycle $\mathrm{S}=20$ ), the lowest $3.4 \%$ at $\mathrm{B}=6 \mathrm{mT}$; frequency $\mathrm{Fs}=32 \mathrm{~Hz}$; duty cycle $\mathrm{S}=$ 12 (mode №3).

When studying the qualitative composition of strawberry fruits at the stage of maturation, attention was paid to the change in the osmotic pressure of cell juice in the cell under the influence of various modes of lowfrequency magnetic pulse treatment of plants. Figure 9 shows a histogram of the distribution of osmotic pressure of cell juice in the cell of strawberry fruits, depending on the treatment modes of plants with a low-frequency magnetic pulse field, which varied from 249 to $355 \mathrm{kPa}$.

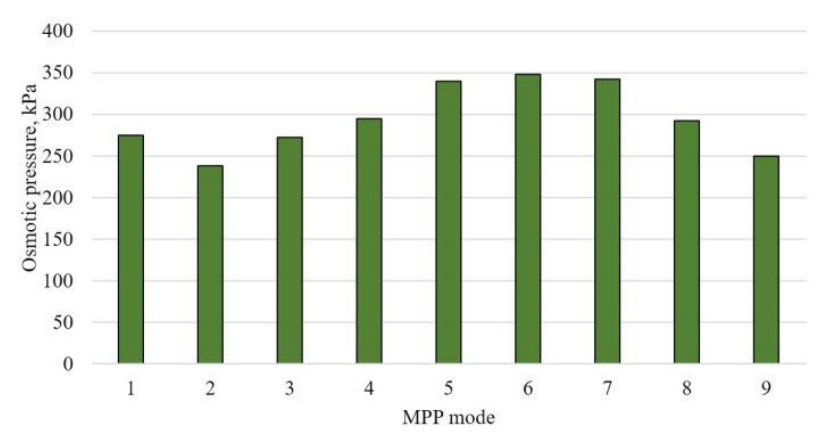

Fig. 9. Osmotic pressure of cell juice in the cells of strawberries in the fruit-ripening phase.

The analysis of the obtained data showed that lowfrequency magnetic pulse treatment of plants led to an increase in the osmotic pressure of the juice in the fruit cells compared to the control. The highest values of the increase in osmotic pressure were obtained when treating plants with modes № 5, 6, 7, in which deviations from the control values were $30 \%, 32.8 \%$ and $30.3 \%$, respectively. Mode parameters: № 5 (induction $\mathrm{B}=6$ $\mathrm{mT}$, frequency $\mathrm{Fs}=32 \mathrm{~Hz}$, duty cycle $\mathrm{S}=20$ ), № 6 (induction $\mathrm{B}=4 \mathrm{mT}$, frequency $\mathrm{Fs}=8 \mathrm{~Hz}$, duty cycle $\mathrm{S}$ = 16), №7 (induction $\mathrm{B}=4 \mathrm{mT}$, frequency $\mathrm{Fs}=32 \mathrm{~Hz}$, duty cycle $\mathrm{S}=12$ ). The largest deviation $(32.8 \%)$ from the control values was obtained when plants were treated with a magnetic pulse field with the following parameters: induction $\mathrm{B}=4 \mathrm{mT}$; frequency $\mathrm{Fs}=8 \mathrm{~Hz}$; duty cycle $\mathrm{S}=16$ (mode №6), which indicates a decrease in the water-retaining power of plants.

\section{Conclusions}

Conducted in a climatic chamber experiment to assess the impact of low-frequency magnetic pulse radiation on plants of the garden strawberry was allowed to draw the following conclusions: installed stimulating mode of low-frequency magnetic-pulse treatment plants of the strawberry cultivar "Malvina", held for 30 days before the formation of stems, with the following parameters: magnetic field induction $\mathrm{B}=4 \mathrm{mT}$; the pulse repetition frequency $\mathrm{Fc}=32 \mathrm{~Hz}$; duty cycle $\mathrm{S}=20$, the impact of which has led to increased compared with the control weight of the aerial parts of plants (leaves) in the phase formation of stems, leaf area, chlorophyll content in leaves, chlorophyll $\mathrm{b}$, carotenoids content, ascorbic acid content in fruits, nitrates. However, the largest amount of sucrose in strawberry fruits at the ripening stage was obtained with the parameters of the processing mode: magnetic field induction $\mathrm{B}=6 \mathrm{mT}$.

Thus, because of the conducted experimental studies, the relationship between the parameters of lowfrequency magnetic pulse radiation and the photosynthetic activity of the leaf apparatus at various stages of ontogenesis, the qualitative composition of fruits at the ripening stage was revealed. The high sensitivity of plant organisms to the effects of energy factors, the parameters of which differ in exposure and physical factors, is shown. At the same time, the nature of the response of plants is complex, ambiguous and is determined not only by the electrophysical parameters of the electric field, but also by the specific, varietal and technological features of the crop.

\section{References}

1. E.M. Massimo, Magnetic field effects on plant growth, development and evolution, Frontiers in Plant Science, 1-13 (2014)

2. J. Ben Ammar, J.L. Lanoiselle, N.I. Lebovka, E. Van Hecke, E. Vorobiev, Impact of a pulsed electric field on damage of plant tissues: Effects of cell size and tissue elec-trical conductivity, Journal of Food Science, 76, E90-E97 (2011)

3. R. Radhakrishnan, B.D.R. Kumari, Pulsed magnetic field: a contemporary approach offers to enhance plant growth and yield of soybean, Plant Physiology and Biochemistry, 51, 139-144 (2012)

4. G. Fischer, M. Tausz, M. Kock, D. Grill, Effects of weak $162 / 3 \mathrm{~Hz}$ magnetic fields on growth parameters of young sunflower and wheat seedlings, Bioelectromagnetics, 25, 638-641 (2004)

5. D.J. Bilalis, N. Katsenios, A. Efthimiadou, A. Karkanis, Pulsed electromagnetic field: an organic compatible method topromote plant growth and yield in two corn types, Electromagn. Biol. Med., 3, 333-343 (2012)

6. I.G. Smirnov, D.O. Khort, R.A. Filippov, A.I. Kutyrev, Factor analysis of irradiation of the strawberries (fragaria $\times$ ananassa) seeds pulsed 
low-frequency magnetic field, Indian Journal of Agricultural Science, 89, 113-118 (2019)

7. A.Y. Izmailov, I.G. Smirnov, D.O. Khort, R.A. Filippov, A.I. Kutyrev, Magnetic-pulse processing of seeds of berry crops, Research in Agricultural Engineering, 4, 181-186 (2018)

8. A. Esitken, M. Turan, Alternating magnetic field effects on yield and plant nutrient element composition of strawberry (Fragaria $\mathrm{x}$ ananassa cv. Camarosa), Acta Agriculturae Scandinavica, 54 (2004)

9. I.G. Smirnov, D.O. Khort, R.A. Filippov, A.I. Kutyrev, A.A. Artiushin, Automated Unit for Magnetic-Pulse Processing of Plants in Horticulture, Mordovia University Bulletin, 28, 624-642 (2018)

10. P. Galland, A. Pazur, Magnetoreception in plants International Journal of Plant Research, 118 (6), 371-389 (2005)

11. V.N. Bingi, Magnitobiology: Experiments and Models (Moscow, MILTA, 2002, 592)

12. H.H. Hsin, W. Show-Ran, The effects of $60 \mathrm{~Hz}$ magneticfields on plant growth, Nature and Science, 1, 60-67 (2007)

13. D.R. Mihaela, C. Dorina, A. Carmen, Biochemical changesinduced by low frequency magnetic field exposure of vegetalorganism, Romanian Journal of Physics, 52 (7), 645-651 (2006)

14. D. Khort, I. Smirnov, A. Kutyrev, R. Filippov, Device for automated magnetic pulse stimulation of plant production processes, E3S Web of Conferences, ICEPP 2020, 01064 (2020)

15. A.I. Kutyrev, D.O. Hort, R.A. Filippov, Y.S. Tsench, Magnetic-pulse treatment of strawberry seeds, Agricultural machines and technologies, 5, 9-15 (2013). 